\title{
Olfaction-based Detection Distance: A Quantitative Analysis of How Far Away Dogs Recognize Tortoise Odor and Follow It to Source
}

\author{
Mary E. Cablk ${ }^{1} *$, John C. Sagebiel ${ }^{2}$, Jill S. Heaton ${ }^{3}$ and Cindee Valentin ${ }^{4}$ \\ ${ }^{1}$ Desert Research Institute, Division of Earth and Ecosystem Sciences, 2215 Raggio Parkway, Reno, \\ NV 89512, USA; E-mail: mary.cablk@dri.edu \\ ${ }^{2}$ Environmental Health and Safety, University of Nevada Reno MS 328, Reno, NV 89557, USA; E- \\ mail: jsagebiel@unr.edu. \\ ${ }^{3}$ Department of Geography, University of Nevada Reno MS 154, Reno, NV 89557, USA; E-mail: \\ jheaton@unr.edu \\ ${ }^{4}$ Applegate School for Dogs, PO Box 5577, Walnut Creek, CA 94596, USA; E-mail: \\ cindeeval@aol.com
}

* Author to whom correspondence should be addressed; E-mail: mary.cablk@dri.edu

Received: 14 January 2008 / Accepted: 27 March 2008 / Published: 28 March 2008

\begin{abstract}
The use of detector dogs has been demonstrated to be effective and safe for finding Mojave desert tortoises and provides certain advantages over humans in field surveys. Unlike humans who rely on visual cues for target identification, dogs use primarily olfactory cues and can therefore locate targets that are not visually obvious. One of the key benefits of surveying with dogs is their efficiency at covering ground and their ability to detect targets from long distances. Dogs may investigate potential targets using visual cues but confirm the presence of a target based on scent. Everything that emits odor does so via vapor-phase molecules and the components comprising a particular scent are carried primarily though bulk movement of the atmosphere. It is the ability to search for target odor and then go to its source that makes dogs ideal for rapid target recognition in the field setting. Using tortoises as targets, we quantified distances that dogs detected tortoise scent, followed it to source, and correctly identified tortoises as targets. Detection distance data were collected during experimental trials with advanced global positioning system (GPS) technology and then analyzed using geographic information system (GIS) modeling techniques. Detection distances ranged from $0.5 \mathrm{~m}$ to $62.8 \mathrm{~m}$ for tortoises on the
\end{abstract}


surface. We did not observe bias with tortoise size, age class, sex or the degree to which tortoises were handled prior to being found by the dogs. The methodology we developed to quantify olfaction-based detection distance using dogs can be applied to other targets that dogs are trained to find.

Keywords: detection distance, olfaction, biosensor, dog, desert tortoise, probability of detection, Mojave Desert.

\section{Introduction}

Mojave desert tortoises (Gopherus agassizii) occur throughout the Mojave Desert; populations north and west of the Colorado River are listed as 'threatened' under the U.S. Endangered Species Act because they are in decline ([1], [2]). The use of dogs to survey for this rare and cryptic reptile has been demonstrated to be safe, effective and cost efficient [3]. Dogs were initially investigated for use as an alternative survey tool to humans, who are limited to visual cues for finding tortoises that spend a majority of their time underground in burrows [4]. The use of dogs for other wildlife detection applications has become more widespread as their utility is demonstrated at detecting targets that are difficult for humans to locate reliably ([5], [6], [7]). Dogs are used to find mammalian scat for population assessment and monitoring in the wild ([8], [9], [10]). Law enforcement contraband dogs are used to detect illegal animals and animal parts smuggled in and out of countries around the world. Dogs are reported in a variety of literature to be used for numerous other wildlife and biological detection purposes including locating bat carcasses under wind generators [11], seeking household mold, pests or diseases on agricultural products [12], off-flavor catfish [13], and invasive weeds [14]. Dogs have been shown to be able to differentiate at least 10 different compounds without degradation in performance and become more efficient as new target compounds are learned [15]. Just how they are able to do this remains unknown and thus how to optimize their performance with search and deployment strategies is likewise unknown.

The vertebrate olfactory system is known to be complex but has yet to be completely described in terms of how odors are recognized in the brain ([16], [17]). Olfaction is an important and significant sense in mammals capable of precise chemosensation [16]. Artificial noses have yet to be created that can match the capability and field utility of an animal's nose. Both artificial noses and dogs return variable responses to repeated encounter with the target stimulus [18], however dogs have the ability to respond to novel odor compounds, to resist damage, to go to source, to detect targets in chemically 'noisy' environments and have detection thresholds not yet matched with instruments [19]. Perhaps most importantly, dogs can generalize; that is, they can learn to identify a discrete set of individuals within a target class and then detect in the field new individuals they have never before encountered. Search dogs trained to find missing people continually demonstrate the ability to generalize. Trained on a certain set of individuals, they find and alert on unfamiliar people, old and young, of different races and in varying states of health, even near-dead or deceased. Similarly where tortoises are the 
target, new individual animals can be of different age, size, sex or potentially state of health and still be located by the dog.

For practical field deployment to locate wildlife or wildlife sign (scat, hair, musk, etc.), the sensor must be able to independently seek the target odor, cover great distances, differentiate between targets that may be similar in odor or share common odor signature elements, lead the observer to and then pinpoint the source of the odor within a large unconstrained search area, identify variable levels of target odor, continuously learn and refine the scent search image, and locate targets that are not surface visible (i.e. under cover or in dens/burrows, under water, in trees, etc.). Artificial noses are not a viable tool for conducting wildlife searches because they cannot meet these criteria, whereas dogs do.

\subsection{Fundamentals of odor detection}

To accurately discuss the ability of dogs to detect a target (in our study a tortoise) by olfaction there is a necessary sequence of events that must occur. First the odor molecule(s) that dogs recognize as "tortoise" must evaporate. Our experience training dogs to find tortoises using gauze wiped over the tortoise neck and front legs suggests that whatever the dogs are using to identify the tortoise can be transferred to the gauze from these parts of the body [20]. An alternate source for tortoise odor could be from the breath, but given that dogs trained on skin transfer items can identify a live tortoise we feel the skin is the most likely source for the scent dogs recognize as 'tortoise'. Once the odor molecule(s) evaporates it must diffuse through the boundary layer next to the tortoise's skin and then move via diffusion and bulk air movement to the dog's nose. Once there, the dog must recognize the odor and determine its source direction, which enables the dog to move towards the source based on increased concentration. The concentration of the tortoise odor at the dog's nose will be a function of both the source strength (evaporation rate) and its movement and dissipation in the air. Source strength is a function of the vapor pressure of the compound, the ambient temperature, and is influenced by any binding or affinity the compounds have for the substrate they are on. We presume here that the compounds comprising tortoise odor are semi-volatile and in low concentration, thus the evaporation rate is effectively independent of the size of the tortoise since the larger surface area is not significant considering the low vapor pressure and low concentration of these compounds on the tortoise surfaces. This presumption is based on the fact that the dogs were trained on residual scent captured in gauze pads. If the tortoise odor molecules were not semi-volatile, the dogs would have been trained to find gauze and would not have recognized live tortoises. The binding or affinity between the skin or other surface, such as vegetation or soil, and the odor compound will be affected by ambient humidity since water can displace odor at binding sites. This effect has been quantified in the interaction between soil and applied pesticides [21] and we suspect a similar effect occurs with tortoises.

Transport of the evaporated odor compounds in the air will be a function of the wind speed and direction and the roughness of the terrain. Scent movement is often described by experienced dog handlers as "pooling" or getting "trapped" in areas. This is a qualitative way of describing the impact of wind and terrain on movement of any chemical in the air under the influence of these effects. At higher wind speeds, scent is transported rapidly down wind, but it also moves vertically and perpendicular to wind direction due to turbulent air movements, thus the resulting scent concentration pattern is described as either a plume or cone of scent with the highest concentration at the mid-line. 
The shape of the plume is influenced strongly by wind speed and roughness of the terrain. At the extreme of no wind, all movement of the compound will be by molecular diffusion and a "pool" of scent will form with concentric rings of decreasing concentration. Concentration is critical since it is clear that at some low concentration the dog is unable to detect the scent which represents a detection limit for the sensor.

\subsection{Sensor deployment and target detection distance}

Search strategy is the means used to maximize target detection. This concept is applied at different scales, for example an incident commander searching for a lost person might employ a search strategy that involves foot searchers, aircraft, and search dog teams. At this level the objective is to maximize resource allocation based on probability of area (POA), the likelihood that the target is in a defined region. At a micro scale, search strategy is the actual pattern a searcher uses to search a vector, such as a vehicle, a building, or a geographic area. Ideally search strategy is optimized to minimize time worked and distance covered while maximizing the number of targets found. Even with an optimized search strategy, target detection ultimately rests on the ability of the search tool whether human, dog, or synthetic sensor. Chemosensor operators also employ search strategy; it can be as simple as placing a sensor in a ventilation return duct to detect compounds in a room or as complex as a systematic grid pattern through a room or area. Search strategy has been investigated for different applications from theoretical [22] to animal predation and foraging [23] to sampling of rare species ([24], [25]) among others [26]. Olfaction-based detection distance by dogs and search strategy are interrelated but the relationship between the two has not been quantified. Detection distance is simply how far away a target is detected, independent of the sensory means employed. In the context of considering a detection dog to be a chemical sensor, detection distance is the point where the dog exceeded its minimum detection limit, since it was able at that moment to detect and identify (recognize) the target source. This is not necessarily the maximum detection limit for those conditions since scent pools vary in shape and size as described above. Therefore, detection distance varies not only with detection threshold of the dog, but also with the physical shape of the odor concentration distribution.

For humans, target detection such as tortoises, scat, missing people, landmines, or buried items to name a few is based primarily on visual or sometimes auditory clues. Efficacy of night searching, searching in low light conditions, or searching in dense vegetation or other type of cover by humans is therefore very limited. Similarly for animals, visual detection ability is affected by the quality of the surrounding medium such as air or water. Visual detection distance has been investigated as a function of light level and turbidity in fish and related predator-prey detection of each other [27]. Such responses can be explored with theoretical models that describe the role of detection distance between predators and prey in escape behavior [28]. One study [29] evaluated the relationship between the distance birds detected a predator and subsequent escape behavior. In detection work, dogs acquire and use olfactory search images [30] and have been shown to rely primarily on olfaction to detect stationary and cryptic or buried objects [31]. Together, detection distance and search strategy affect the probability of detection (POD), which is the likelihood that a target will be located. In detection dog disciplines POD is reported as a percentage. For wildlife detection applications, where large areas or long distances are to be searched, it is important to first determine POA based on best available 
information about the species and its habitat, and subsequently within the selected search areas determine a search strategy to achieve the desired POD. Although a 100\% POD is always desirable, in the field setting it is not a realistic expectation ([20], [10]).

Studies on the effectiveness of dogs in the field setting have returned varying results [15], but dogs have been shown to be quite capable of detecting targets under natural search conditions ([32], [3], [33]). Experimental results from data collected by dogs vary due to the quality of protocols used to train the dogs and the capabilities of personnel involved in the research. It is particularly challenging to accurately design and execute a research study where dogs are trained for olfactory investigations and then used to collect data. Dogs learn independently, often picking up on subtle clues from their handlers or observers who are unaware they are influencing the dogs' responses [34]. Dogs are also exceedingly more sensitive than instrumentation, thus great care is required to prevent cross contamination of target and non-target material. Also as a result of the dog being more sensitive than instrumentation and better at detecting certain targets than humans is the fact that the dog's correct response cannot always be validated by humans, either quickly if at all. Finally, researchers are typically not trained nor do they necessarily understand that these behavioral and physical sensitivities exist [35]. It is easy to inadvertently train a dog to detect an unexpected or impure source when attempting to train to a pure compound. This error is amplified in the field setting where the complexities of dog-handler dynamics, terrain, weather, 'noisy' odor conditions, and stress affect dog performance.

Finally, the need exists to conduct studies quantifying POD for dogs searching an outdoor, natural environment. Search behavior in a laboratory setting has been evaluated for rats [36] and researchers at Sokoine University of Agriculture in conjunction with the University of Antwerp have developed grid search strategies for rats trained to detect buried landmines in the field setting [37]. Given that dogs were shown to be effective and reliable at finding Mojave desert tortoises we sought to devise a methodology that would capture the fine detail of a dog working to target source for the purpose of determining what an expected range of detection distances for Mojave desert tortoises on the landscape surface (as opposed to in burrows underground) might be. Ultimately this methodology and resulting information could be used to refine search strategy and reported POD during actual surveys and in other search and detection operations.

\section{Experimental Section}

The research was conducted 1-28 April 2004 at the Desert Tortoise Conservation Center near Las Vegas, NV, USA. All research was conducted with the appropriate permits and with permission of the U.S. Bureau of Land Management who manages the research facility. This study was part of a larger proof-of-concept study evaluating the use of dogs to find desert tortoises and a full description of the experimental design for the larger research project can be read in [20]. In summary, between 2 and 4 live desert tortoises were tethered at random locations in each of 26 different survey plots of either 0.5 ha or 2 ha in size. Two different dog teams, consisting of one dog working off-leash and one handler, searched the plots for tortoises. The handlers were not provided any information about the number of tortoises tethered or their location. When a dog found a tortoise it performed its trained alert (a 'sit') (Figure 1). Dog teams searched trial plots in a single pass with no backtracking or time limit 
restrictions. Handlers determined their search strategy to best cover their assigned area based on wind conditions and terrain. These protocols were employed to simulate actual field survey conditions.

Figure 1. A tortoise detection dog performs his trained alert, the 'sit', near a tortoise in training. The dog demonstrates his ability to perform an independent alert upon locating and confirming the target.

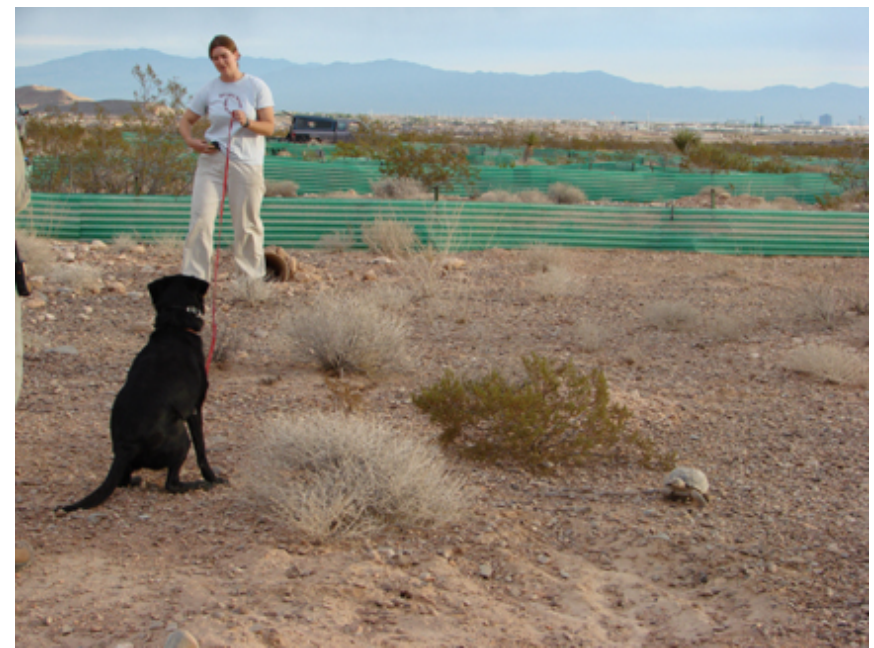

Figure 2. Tortoise detection dogs carried GPS units that collected data in 1-s increments. The GPS units were placed in dog vests fitted with pockets. The data were post-processed to have cm-level geographic positional accuracy.

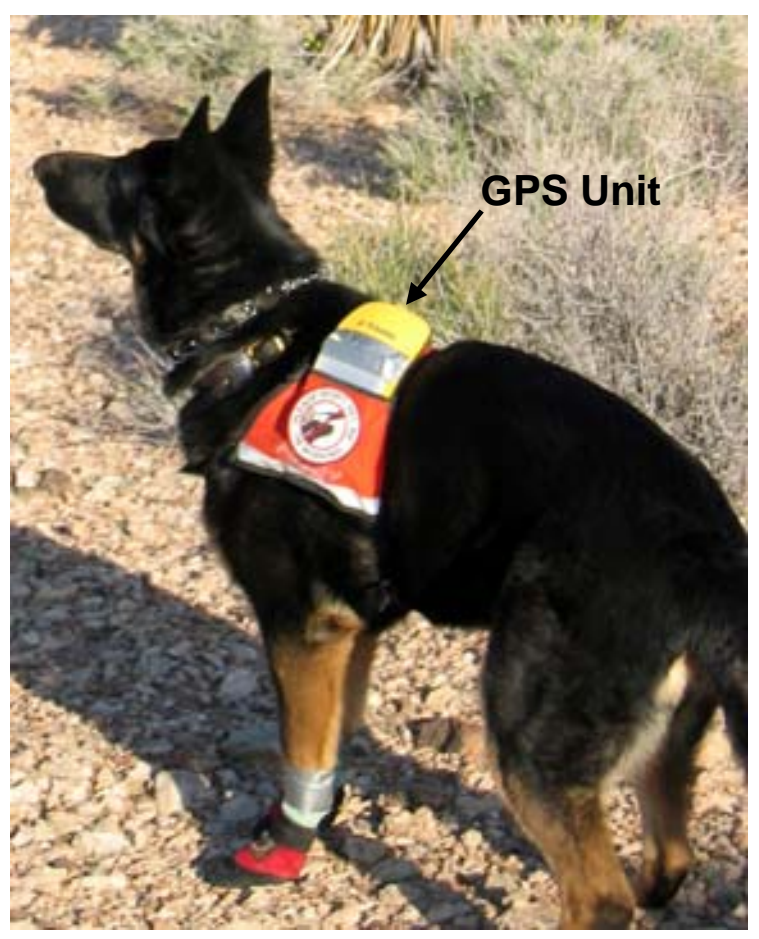

Dog and handler movements were recorded using global positioning systems (GPS) in 1-second increments (Figure 2) and data were post processed to centimeter-level accuracy. These data allowed identification of the exact point at which the dog changed direction and moved directly towards a 
tortoise. The distance between change of direction and tortoise location was the calculated detection distance, analyzed using the proximity point tool in ESRI ArcGIS. Each change in direction towards a tortoise was evaluated against recorded environmental conditions to determine if the find was likely based on wind-carried scent or due to visual observation by the dog. If the dog were upwind of a tortoise it was assumed to have seen the tortoise rather than smelled it. Four weather stations were evenly distributed within each plot and collected wind speed and direction and temperature data at approximately $0.5 \mathrm{~m}$ off the ground; one of the four also recorded relative humidity. The weather station data loggers collected data in 1-second increments synchronized with the GPS units.

\section{Results and Discussion}

The dogs encountered many more tortoises than were tethered as part of the research trials because tortoises not involved in our study lived in the pens where our trials were conducted. While we quantified some of the variables that may influence target variants it is not necessary to capture all variants to assess detection distance. As a target, tortoises present greater variability in scent than single molecule target compounds such as cocaine, for example. For the detection distance calculation, whether or not a tortoise was in a trial was irrelevant so long as the find was verified. The total number of tortoises encountered by the dogs, both tethered and wild, was 184 . The total number missed from the known placements was 8 . Of these 184 encounters, we believe the dogs first detected 21 tortoises visually for a total data set of 163 most likely detected by olfaction. Visual detections were ascertained by the dog GPS track data showing direct movement to a tortoise while upwind. Of the 163 tortoise encounters, 99 were part of our study and were measured and sexed ('known'). The other 64 tortoises were 'wild' and we did not take measurements of these animals. Size of the known tortoises thus located $(n=99)$ were between $89 \mathrm{~mm}$ and $300 \mathrm{~mm}$ midline carapace length (MCL; Table 1). Detection distances were evaluated for normality to determine whether parametric or nonparametric statistical tests were appropriate. The data were non-normally distributed and had positive and significant values of skewness $(1.7552, \mathrm{p}<0.0001)$ and kurtosis $(3.4910, \mathrm{p}<0.0001)$, respectively.

Table 1. Summary statistics of detection distances for surface tortoises located using air scent during trials. "Known" were tortoises tethered as part of the study $(\mathrm{n}=99)$. "Wild" tortoises were those that were located by the dogs but not placed as part of the study $(n=64)$. "All" summarizes all tortoises located by the dogs during the study $(\mathrm{n}=163)$.

\begin{tabular}{|l|l|l|l|}
\hline & Known $(\mathrm{m})$ & Wild $(\mathrm{m})$ & All $(\mathrm{m})$ \\
\hline Minimum & 0.75 & 0.50 & 0.50 \\
\hline Maximum & 56.01 & 62.82 & 62.82 \\
\hline Mean & 13.31 & 14.84 & 13.91 \\
\hline Median & 11.24 & 10.35 & 11.13 \\
\hline
\end{tabular}

For all tortoise encounters, detection distance ranged between $0.50 \mathrm{~m}$ and $62.82 \mathrm{~m}$ with a median of $11.13 \mathrm{~m}$ (mean of $13.91 \mathrm{~m}$ ). We found no difference in detection distances between the dogs (Kruskall-Wallis 1-way ANOVA; $n=163, \mathrm{p}=0.7594$ ) for either type of tortoise encounter, wild or 
known. We found no difference in detection distances by sex of the tortoise $(p=0.2862)$, by size class $(\mathrm{p}=0.5350)$ or by individual tortoise $(\mathrm{p}=0.6859)$. Tortoise size and age are generally positively related. From a deployment and sampling perspective, it is important to note that there was no sex or size bias in detectability of the tortoises. There did not appear to be any single tortoise that the dogs learned to find or had a particular attraction to over others. There was no significant relationship between the time of day and detection distance $(r=0.03, p=0.7199)$ and detection distances in each trial were not significantly different $(n=163, p=0.0157)$, although environmental conditions between trials (relative humidity, temperature, wind speed means) were significantly different. Detection times ranged between 0745 and 1753 . The dogs were able to detect tortoises from variable distances with a level of equal proficiency [20] despite variable environmental conditions that are thought to affect scent behavior and so affect vapor transport.

Temperatures $(\mathrm{T})$ recorded during the trials ranged from $12 \mathrm{C}-27 \mathrm{C}$. Relative humidity $(\mathrm{RH})$ ranged between $16 \%$ and $85 \%$. Wind speed ranged between $0 \mathrm{~m} / \mathrm{s}$ and $9 \mathrm{~m} / \mathrm{s}$. Pearson correlation results for detection distance and mean humidity during the trials was negative and significant $(\mathrm{r}=$ 0.33 , 2-tailed $\mathrm{p}<0.0001)$ and was significant and positively related to both mean temperature $(\mathrm{r}=$ $0.35,2$-tailed $\mathrm{p}<0.0001)$ and mean wind speed $(\mathrm{r}=0.27,2$-tailed $\mathrm{p}=0.0005)$. The greater the wind speed, the greater the detection distance. Given our understanding of the processes necessary for a dog to detect tortoise odor, we know that higher temperatures will cause more evaporation and higher winds will transport the odor farther. This analysis is complicated by the inter-relationships among $\mathrm{T}$, RH and wind speed. As expected, when T increased wind speeds increased and RH dropped (Table 2). It is likely that there was insufficient variation in climatic conditions during this study (e.g. a day with high RH and high temperature) to fully separate the influence of these parameters. RH is more challenging to interpret; since we do not know exactly what the tortoise odor compounds are we cannot be sure how RH influences their behavior.

Table 2. Correlation coefficients for wind speed $(\mathrm{m} / \mathrm{s})$, temperature $(\mathrm{C})$ and humidity.

\begin{tabular}{|l|l|l|l|}
\hline & \multicolumn{1}{|c|}{ Wind speed } & \multicolumn{1}{c|}{ temperature } & \multicolumn{1}{c|}{ humidity } \\
\hline Wind speed & 1.0000000 & 0.4238679 & -0.4888721 \\
\hline Temperature & 0.4238679 & 1.0000000 & -0.9172985 \\
\hline Humidity & -0.4888721 & -0.9172985 & 1.0000000 \\
\hline
\end{tabular}

The results of the detection distance analysis showed that dogs detected surface tortoises from distances $\geq 60 \mathrm{~m}$ and $\leq 1 \mathrm{~m}$. Detection distance was independent of size or sex of tortoise and whether or not the tortoise had epoxy, glue, or a transmitter. The dogs were able to learn tortoise scent through extraneous anthropogenic scent and located them from long distances regardless of the tortoise's level of human handling. This is an important factor because dogs would be expected to encounter tortoises with and without transmitters in the field during actual surveys. Based on our results there is no reason to expect detection distance bias in surveys using dogs from tortoises with or without hardware or prior human handling. Dogs can detect as well as differentiate tortoise odor from all other non-tortoise scent. In fact, the dogs demonstrated that they could differentiate live tortoises from scat and from tortoise urine, as the dogs never alerted on tortoise scat or urine during the trials. Few carcasses were 
encountered during the study and dogs did not alert on the few encountered, nor did the dogs indicate falsely on other flora or fauna.

\subsection{Quantifying detection distance - a new methodology}

Both search and rescue and detection dog handlers who work dogs off-leash over large areas have observed and describe sudden changes in direction of the dog's nose followed by a subsequent change in the dog's direction of travel. This is believed to occur when the dog enters what could be envisioned as a line of detectable scent that the dog identifies as its trained target odor (Figure 3). Because this behavior was qualitatively described by search dog handlers but scientifically undocumented and because nothing was known about tortoise scent, it was unclear whether or not the methodology used in this study would allow us to obtain data for detection distance analyses. The use of GPS location data collected at the highest repeat increment possible (1-second) and the ability to differentiallycorrect the data to centimeter level accuracy of both the dog movement and the tortoise location was imperative to achieve accurate results. Even with WAAS enabled (Wide Area Augmentation System), uncorrected GPS data are not accurate enough to determine precise detection distances, primarily because both detection distances and the moment of change in direction towards the target source could be within the horizontal error of the GPS unit.

A GPS that collects real-time differentially corrected data, which downloads both the GPS signals and the correction factors and then applies the geometric corrections internally is ideal although post processing data will also provide accurate track data. At present such GPS units are costly and are relatively large and heavy. To prevent physical injury to the dog from wearing a GPS the unit should be counter-balanced. This also keeps the GPS antennae pointed upward to maximize continuity in satellite reception. Dogs need to be comfortable with and accustomed to working while wearing equipment for detection distance data to yield valid results. Desensitizing working dogs to such equipment is simple and does not require much time. These fine details are important to ensure that the dogs are working uninhibited by equipment or experiencing distress. Either of these factors may affect their responses and thus affect the detection distance data.

\subsection{What comprises 'tortoise' scent that dogs recognize?}

In this study the tortoises were targets for the detector (the dog). As a target, tortoises are inherently more complex and more variable than a single molecule compound. While tortoises represent a more variable scent than cocaine for example, for the purposes of this study we consider tortoises to be a single source that our detector, the dog, can identify in the natural environment. Exactly what constitutes 'tortoise' scent that the dogs cue on remains unidentified and until this is solved we will not be able to quantify precisely the source strength, i.e., the rate of evaporation of these compound or compounds from the tortoise. Trained dogs can detect tortoises in burrows ([20], [3]) which suggest that the detection is from vapor phase compounds that can diffuse outward; this is consistent with scent theory that states only vapor phase compounds can be smelled. 
Figure 3. Handler and dog tracks and tortoise locations for one trial. The GPS captured what is often called a 'head snap', where the dog makes a sudden sharp change in direction off his/her trajectory and goes directly to source.

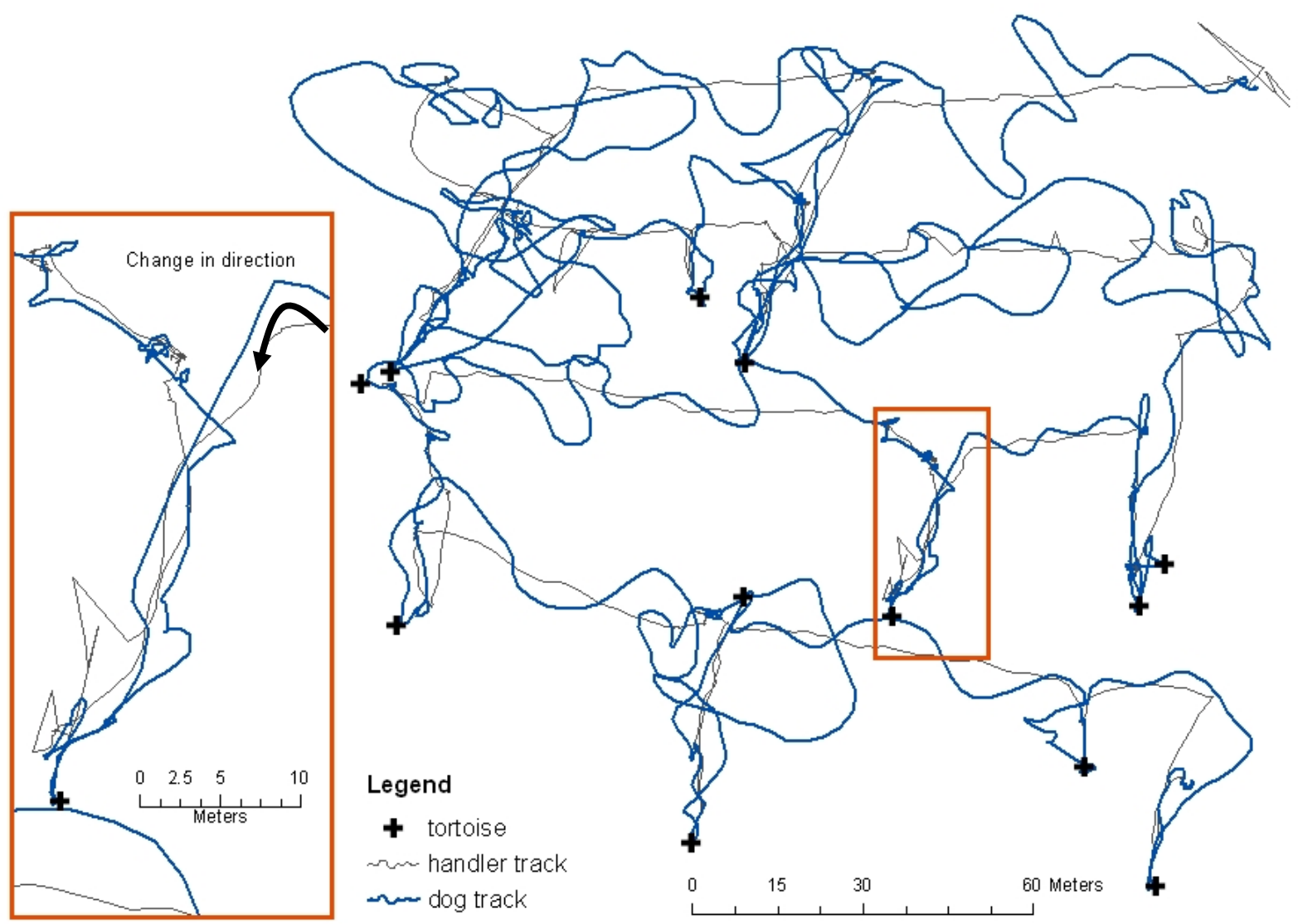

Source strength is one variable used in the field setting to predict detection distances for optimizing search strategy in that the stronger the source, the more vapor is available to be transported. The second major variable that affects detection distance is the odor transport and dispersion by air movement. Therefore determining a search strategy based upon predicted or expected detection distances directly affects a handler's POD. Probability of detection is an important variable reported by dog handlers in working dog disciplines and for wildlife detection because it directly affects study and monitoring results. A higher POD results in a higher likelihood that all targets are found, and thus more samples (data) are collected; so in the case of presence/absence surveys, the POD can directly affect determinations about whether or not a species exists in the survey area. Reporting POD is the numerical equivalent of answering the question, "How confident are you that you that you found all of the targets"? For tortoises two studies have quantified POD (efficacy) for detection using known populations ([3], [20]).

The variable detection distances measured in our study may have been affected by differing source strengths of individuals that were not captured in our sample size or sex categories; however, it is equally likely that the evaporation rate is slow enough that tortoise size has an insignificant effect. We compared detection distances with environmental data and concede artificially imposed limitations on detection distance dictated by our study plot sizes. Although we did not find a relationship between 
detection distance and any tortoise-specific variable measured (age class, sex, size) some of the variability seen in our measured detection distances may be attributable to individual tortoises rather than environmental conditions. It would be worthwhile to conduct experimental trials to quantify detection distances under a greater range of environmental conditions as well as investigations into individual tortoise scent variability.

\subsection{Training on limited variants and generalization}

Search and detection dog handlers are keenly aware that while dogs are able to generalize from the few individual targets they are trained on to new and unfamiliar variants, the more target variants the dogs are trained on, the more unfamiliar variants they will correctly identify [19]. During this study the dogs were trained on, or learned through encounters in trials, tortoises that were at least $89 \mathrm{~mm}$ in length, called juveniles, up to large adults. After the detection distance trials were completed, we conducted some additional trials that are not included in this study [38]; during these additional trials the dogs found five very small $(30,40,50,50$ and $80 \mathrm{~mm})$ tortoises on the surface using olfaction. Dogs were observed to follow scent to these tiny tortoises and perform their trained alert, indicating they recognized the small tortoises as belonging to the general class 'tortoise'. This is significant because the dogs had not been trained on tortoises this small yet they were able to correctly recognize them as being 'tortoise'. We cannot say how many small tortoises (i.e. $<90 \mathrm{~mm}$ ) were present in any of the pens in which dogs were fielded, so we cannot say how many small tortoises the dogs missed. However, we can report that at a certain point (be it after a set number of encounters with reward, after small tortoises began to surface, or due to specific weather conditions, none of which with we can cite with any certainty) the dogs generalized small tortoises; this further lends evidence to our theory that evaporative rate of tortoise odor is effectively independent of tortoise size.

Another important result of this work is the demonstration of dogs' abilities to be trained on residual scent from a relatively small number of live tortoises kept in captivity in a highly unnatural environment, fed a non-natural diet and maintained in unnatural enclosures with unnatural bedding material - and then go on to find completely different and unrelated live tortoises maintained outdoors in their native habitat with minimal to no subsidy by humans. This also demonstrates the dogs' ability to generalize. Each encounter with a new tortoise over the course of the research trials increased the dogs' pool of variants and generalization is enhanced with exposure to more variants [19]. This finding lends support to the validity of collecting scent articles or training aids through residual scent transfer in other detection dog disciplines.

\subsection{The role of scent pool morphology in field deployment}

It is important to acknowledge scent cone or scent pool morphology in the field setting. Scent cone shape, size, and persistence cannot be definitively delineated although these properties can be observed through olfactory-behavioral responses of dogs in target odor. The detection distances calculated here cannot be interpreted as maximum distance of dog finds or maximum detection distance under a set of environmental conditions because we have no way of determining physically where in the scent cone the dogs hit their minimum detection threshold. They may have encountered it at the farthest downwind range of the cone or may have encountered it in the middle. We recommend further studies 
with larger study sites to better understand the relationships between detection distance and the environmental and tortoise-related variables we measured.

\subsection{Practical applications of our results}

Although the range of environmental conditions during the trials was a subset of the broader range that occurs in the Mojave Desert, conditions were representative of those during which tortoises are active in the spring and fall ([2], [3]). It is not currently possible to extrapolate our detection distance results to tortoises in burrows or to small tortoises less than $100 \mathrm{~mm}$ MCL since we have no data representing either of these categories, although based on our observations from [38], scent transport and detectability of small tortoises on the surface should be consistent with our findings here. The movement of vapor from tortoise burrows into open air is unstudied and to assume that scent would be transported as readily or in the same manner as from surface tortoises is simply too great a leap to make. However, we have observed dogs perform alerts at the mouth of occupied burrows ([3], [20]) that suggests burrow screening by dogs is another valid and useful application. Finally, the method we developed was successful for determining detection distances and could be applied for other wildlife, wildlife sign or other targets.

\section{Conclusions}

Dogs were shown to generalize to live Mojave desert tortoises when initially trained on a discrete set of tortoise residual scent training aids. During the study reported here, dogs trained on adult tortoises went on to find new individuals not previously encountered, which is consistent with other dog search and detection disciplines. They also located very small tortoises suggesting that tortoise odor across size classes is effectively consistent in its evaporative rate, thus source strength for both small and large tortoises may be similar if not equal. Dogs' abilities to find very small tortoises in natural settings during actual surveys remain undemonstrated and merits further study.

Dogs detected and located tortoises at a range of distances that result from the detection threshold of the dog and the physical location of where the dog encountered that threshold concentration in the scent pool. Detection threshold of the dog is immeasurable until the molecular composition of 'tortoise' scent can be described. The method we developed to measure detection distance can be applied for any target that can be held stationary and visually hidden. The results presented here are directly applicable when developing a dog search strategy in the field with respect to grid spacing and are applicable to expected and reported POD.

\section{Acknowledgements}

We wish to thank the University of Redlands and specifically students Rich Inman, Steve Francis and Ben Yetman who were instrumental in data collection. Alice Whitelaw and Aimee Hurt participated as dog handlers. We thank Michelle McDermott for her assistance with tortoises at the Desert Tortoise Conservation Center and the US Fish and Wildlife Service Desert Tortoise Recovery Office for their support of the project. This work was conducted under USFWS permit TE-073505 and Animal Care and Use Protocol \#A3/04-34. 


\section{References and Notes}

1. U.S. Fish and Wildlife Service. Desert Tortoise (Mojave Population) Recovery Plan. U.S. Fish and Wildlife Service, Portland, Oregon, 1994, 73 pages plus appendices.

2. U.S. Fish and Wildlife Service. Range-wide monitoring of the Mojave Population of the Desert Tortoise: 2001-2005 Summary Report. Reported by the Desert Tortoise Recovery Office, U.S. Fish and Wildlife Service, Reno, Nevada, 2006, 85 pages.

3. Nussear, K.N.; Esque, T.C.; Heaton, J.S.; Cablk, M.E.; Drake, K.K.; Valentin, C.; Yee, J.L.; Medica, P.A. Are wildlife detector dogs or people better at finding tortoises? Journal of Herpetological Conservation and Biology 2008, 3, 103-115.

4. Nagy, K.A.; Medica, P.A. Physiological ecology of desert tortoises in southern Nevada. Herpetologica 1986, 42, 73-92.

5. Vice, D.S.; Engeman, R.M. Brown tree snake discoveries during detector dog inspections following super typhoon Paka. Micronesica 2000, 33, 105-110.

6. Harrison, R.L.A comparison of survey methods for detecting bobcats. Wildlife Society Bulletin 2006, 34, 548-552.

7. Rolland, R.M.; Hamilton, P.K.; Kraus, S.D.; Davenport, B.; Gillett, R.M.; Wasser, S.K. Faecal sampling using detection dogs to study reproduction and health in North Atlantic right whales (Eubalaena glacialis). Journal of Cetacean Research and Management 2006, 8(2),121-125.

8. Akenson, J. J., Henjum, M. G., Wertz, T.T. and Craddock, T.J. Use of dogs and mark-recapture techniques to estimate American black bear density in northeastern Oregon. Ursus, 2004, 12, 203-210.

9. Smith, D.A.; Ralls, K.; Hurt, A.; Adams, B.; Parker, M.; Davenport, B.; Smith, M.C.; Maldonado, J. E. Detection and accuracy rates of dogs trained to find scats of San Joaquin kit foxes (Vulpes macrotis mutica). Animal Conservation 2003, 6, 339-346.

10. Wasser, S.K.; Davenport, B.; Ramage, E.R.; Hunt, K.E.; Parker, M.; Clarke, C.; Stenhouse, G. Scat detection dogs in wildlife research and management: application to grizzly and black bears in the Yellowhead Ecosystem, Alberta, Canada. Canadian Journal of Zoology - Revue Canadienne de Zoologie 2004, 82(3), 475-492.

11. Arnett, B. Use of dogs to recover bat and bird fatalities at wind farms. in E. B. Arnett, technical editor, Relationships between bats and wind turbines in Pennsylvania and West Virginia: an assessment of bat fatality search protocols, patterns of fatality, and behavioral interactions with wind turbines. A final report submitted to the Bats and Wind Energy Cooperative. Bat Conservation International. Austin, Texas, USA, pp. 117 - 124, 2005.

12. Brooks, S.E.; Oi, F.M.; Koehler, P.G. Ability of canine termite detectors to locate live termites and discriminate them from non-termite material. Journal of Economic Entomology 2003, 96(4), 1259-1266.

13. Shelby, R.A.; Schrader, K.K.; Tucker, A.; Klesius, P.H.; Myers, L.J. Detection of catfish offflavour compunds by trained dogs. Aquaculture Research 2004, 35(9), 888-892.

14. Goodwin, K.; Sheley, R.L.; Lynch, W.; Menalled, F. Detection of spotted knapweed (Centaurea maculosa Lam.) using canine olfaction. [abstract] Society for Range Management, Paper No. 131, 2005. 
15. Williams, M.; Johnston, J.M. Training and maintaining the performance of dogs (Canis familiaris) on an increasing number of odor discriminations in a controlled setting. Applied Animal Behaviour Science 2002, 78(1), 55-65.

16. Oldender, T.; Fuchs, T.; Linhart, C.; Shamir, R.; Adams, M.; Kalush, F.; Khen, M.; Lancet, D. The canine olfactory subgenome. Genomics 2004, 83, 361-372.

17. Wilson, D.A.; Stevenson, R.J. Olfactory perceptual learning: the critical role of memory in odor discrimination. Neuroscience and Behavioral Reviews 2003, 27, 307-328.

18. Alkasab, T.K.; Bozza, T.C.; Cleland, T.A.; Dorries, K.M.; Pearce, T.C.; White, J.; Kauer, J.S. Characterizing complex chemosensors: information-theoretic analysis of olfactory systems. Trends in Neurosciences 1999, 22(3), 102-108.

19. Johnston, J.M. Canine capabilities: Operational implications of recent R \& D findings. Institute for Biological Detection Systems, Auburn University, June 1999.

20. Cablk, M.E.; Heaton, J.S. Accuracy and reliability of dogs in surveying for desert tortoise (Gopherus agassizii). Ecological Applications 2006, 16(5), 1926-1935.

21. Majewski, M.S.; McChesney, M.M.; Seiber, J.N. A field comparison of two methods for measuring DCPA soil evaporation rates. Environmental Toxicology and Chemistry 1991, 10, 301-311.

22. Moreau, M.; Benichou, O.; Loverdo, C.; Voituriez, R. Intermittant search processes in disordered medium. EPL 2007, 77(2).

23. Lima, S.L. Putting predators back into behavioral predator-prey interactions. Trends in Ecology and Evolution 2002, 17(2), 70-75.

24. Smith, D.R. Survey design for detecting rare freshwater mussels. Journal of the North American Benthological Society 2006, 25(3), 701-711.

25. Gompper, M.E.; Kays, R.W.; Ray, J.C.; Lapoint, S.D.; Bogan D.A.; Cryan, J.R.. A comparison of noninvasive techniques to survey carnivore communities in Northeastern North America. Wildlife Society Bulletin 2006, 34(4), 1142-1151.

26. Wang, R.; Nickerson, J.V. Search strategy optimization for intruder detection. IEEE Sensors Journal 2007, 7(3), 315-316.

27. Mazur, M.M.; Beauchamp, D.A. A comparison of visual prey detection among species of piscivorous salmonids: effects of light and low turbidities. Environmental Biology of Fishes 2003, 67(4), 397-405.

28. Broom, M.; Ruxton, G.D. You can run - or you can hide: optimal strategies for cryptic prey against pursuit predators. Behavioral Ecology 2005, 16(3), 534-540.

29. Lind, J.; Hollen, L.; Smedberg, E.; Svensson, U.; Vallin, A.; Jakobsson, S. Detection distance influences escape behaviour in two parids, Parus major and P-caeruleus. Journal of Avian Biology 2003, 34(3), 233-236.

30. Gazit, I.; Goldblatt, A.; Terkel, J. Formation of an olfactory search image for explosives odours in sniffer dogs. Ethology 2005, 111(7), 669-680.

31. Gazit, I.; Terkel, J. Domination of olfaction over vision in explosives detection by dogs. Applied Animal Behaviour Science 2003, 82(1), 65-73.

32. Schoon, G.A.A. The effect of the ageing of crime scene objects on the results of scent identification line-ups using trained dogs. Forensic Science International 2005, 147, 43-47. 
33. Komar, D. The use of cadaver dogs in locating scattered, scavenged human remains: Preliminary field test results. Journal of Forensic Sciences 1999, 44(2), 405-408.

34. Bentosela, M.; Mustaca, A.E. Communication between domestic dogs (Canis familiaris) and humans. Revista Latinoamericana de Psicologia 2007, 39(2), 375-387.

35. Walker, D.B.; Walker, J.C.; Cavnar, P.J.; Taylor, J.L.; Pickel, D.H.; Hall, S.B.; Suarez, J.C.. Naturalistic quantification of canine olfactory sensitivity. Applied Animal Behaviour Science 2006, 97, 241-254.

36. Otto, J.; Brown, M.F.; Long, W. Training rats to search and alert on contraband odors. Applied Animal Behaviour Science 2002, 77(3), 217-232.

37. Verhagen, R.; Weetjens, F.; Cox, C.; Weetjens, B. Rats to the rescue: Results of the first tests on a real minefield. Journal of Mine Action 2006, 9(2).

38. Cablk, M.E.; Heaton, J.S. Efficacy and reliability of dogs for surveying desert tortoises: Results from the DT-K9 trials, March 28-April 15, 2004. Final report to U.S. Army Research Office. 57 pp. 2005.

C 2008 by MDPI (http://www.mdpi.org). Reproduction is permitted for noncommercial purposes. 